\section{Terapias convencionales para el cáncer de próstata localizado: prostatectomía versus vigilancia activa}

\section{Conventional therapies for localized prostate cancer: prostatectomy vs active surveillance}

Alicia María Negrete Cruz
Recibido: octubre 2017

Aceptado: octubre 2017

\begin{abstract}
Correspondencia
Dra. Alicia María Negrete Cruz

aliin_nc@hotmail.com

Este artículo debe citarse como

Negrete-Cruz AM. Terapias convencionales para cáncer de próstata localizado: prostatectomía versus vigilancia activa. Rev Mex Urol. 2017 nov-dic;77(6):433-436.

DOI: https://doi.org/10.24245/revmexurol.v77i6.1698
\end{abstract}

En la actualidad el cáncer de próstata representa un problema de salud relevante en la población masculina mexicana por su alta incidencia y mortalidad. El cáncer de próstata es más frecuente que el de pulmón y colon; además, constituye la primera causa de mortalidad por cáncer en adultos, mientras que en Estados Unidos es la tercera. ${ }^{1}$ Aproximadamente 1 de cada 7 hombres es diagnosticado con cáncer de próstata en el transcurso de la vida. Conforme aumenta la edad se incrementa el riesgo de padecerlo. Para el año 2017, la Sociedad Americana contra el Cáncer estima que en Estados Unidos se diagnosticarán alrededor de 161,360 casos nuevos de cáncer de próstata y se reportarán 26,730 muertes por esta neoplasia. $^{2}$

Casi todos los tumores de próstata corresponden a adenocarcinomas y aunque existen otros tipos, son menos frecuentes (sarcomas, carcinomas de células pequeñas, tumores neuroendocrinos, carcinomas de células transicionales). En términos generales, se considera un tumor de crecimiento lento, con evolución de 5 a 15 años para alcanzar un volumen detectable, incluso los síntomas suelen manifestarse en etapas avanzadas de la enfermedad. ${ }^{3}$

Como ocurre en todas las neoplasias, mientras más temprano se establezca el diagnóstico más efectivas serán las intervenciones terapéuticas y mejor el pronóstico del paciente. Para ello existen dos pruebas de detección principales, que pueden alterarse cuando el paciente aún no manifiesta síntomas: 1) determinación del antígeno prostático específico mediante la obtención de sangre, cuyo resultado mayor de $10 \mathrm{ng} / \mathrm{dL}$ sugiere una posibilidad de $50 \%$ de padecer cáncer de próstata, y 2) examen digital del recto; ambas pruebas son accesibles y económicas. Se recomienda realizar estos exámenes anualmente, a partir de los 50 años o después de los 40 años de edad, en caso de antecedente familiar de la neoplasia. ${ }^{2}$ Por tanto, surge la pregunta ¿Cuánto 
estamos interviniendo en la parte preventiva o detección temprana de la enfermedad?

Una vez detectada la alteración en alguna de estas pruebas, o en ambas, deben solicitarse estudios para confirmar el diagnóstico de cáncer y establecer la estadificación. Subsecuentemente se evalúan las características del paciente y se valora el mejor protocolo de tratamiento, por ejemplo:

- Vigilancia activa: consiste en la observación minuciosa de la neoplasia, que incluye citas médicas para realizar ambas pruebas de detección temprana cada seis meses. Si éstas resultan alteradas se solicitan exámenes adicionales o se inicia el protocolo de tratamiento. El objetivo se basa en indicar tratamiento exclusivamente a los pacientes con riesgo de padecer la enfermedad y en evitar sus complicaciones.

- Espera en observación: es un seguimiento menos intensivo y con menos pruebas, basado en la identificación de síntomas específicos para iniciar medidas terapéuticas.

- Prostatectomía radical: consiste en la extirpación total de la glándula prostática y una porción del tejido que le rodea, incluidas las vesículas seminales. Esta modalidad de tratamiento se establece cuando el cáncer se encuentra confinado a la próstata.

- Radioterapia: se indica como tratamiento inicial para el cáncer confinado en la glándula prostática, clasificado en bajo grado; como tratamiento inicial en combinación con terapia hormonal para metástasis; recurrencia después de la cirugía y en pacientes con cáncer en etapa avanzada, con la finalidad de limitar su crecimiento y disminuir los síntomas.
- Crioterapia: se indica en pacientes con cáncer en etapa inicial o en caso de recurrencia después de la radioterapia.

- Terapia hormonal: se indica cuando el tumor se ha propagado a otros tejidos, en casos de recurrencia después de la cirugía o radioterapia. Se administra en forma conjunta con radioterapia para potenciar su eficacia.

- Quimioterapia: se considera en caso de propagación a otros tejidos, cuando la terapia hormonal no es eficaz.

- Vacuna Sipuleucel-T: se aplica en pacientes con cáncer de próstata avanzado sin reacción a la terapia hormonal inicial, en sujetos asintomáticos o con síntomas mínimos.

Estos protocolos deben indicarse con cautela, pues algunos estudios han señalado resultados ambiguos cuando comparan la observación versus intervención quirúrgica para el cáncer de próstata en etapa inicial. De igual manera existe poca evidencia que respalde la comparación entre vigilancia activa con otros tratamientos, aunque algunos estudios preliminares demuestran que sólo un tercio de los pacientes sometidos a esta modalidad terapéutica necesita recibir radiación o cirugía. ${ }^{3}$

Un estudio de cohorte publicado en 2017, realizado por Wilt y sus colaboradores ${ }^{4}$ en 731 pacientes de diferentes sitios del Departamento de Veteranos y el Instituto Nacional del Cáncer, con cáncer de próstata localizado, diagnosticado de noviembre de 1994 a enero de 2002, en el que asignaron al azar para pertenecer al grupo de prostatectomía radical u observación (criterios de inclusión: pacientes ideales para prostatectomía radical, confirmación del tumor por histología, con diagnóstico de cáncer de próstata localizado [estadio T1-T2 NxM0] en el tumor-nodo-metástasis; cáncer de próstata de 
cualquier grado diagnosticado en los últimos 12 meses; concentración de antígeno prostático específico menor de $50 \mathrm{ng} / \mathrm{mL}$, edad máxima de 75 años, con resultados negativos en la gammagrafía ósea para metástasis y esperanza de vida de 10 años), demostró como variable principal el tiempo de seguimiento mínimo de 12 y máximo de 19.5 años o hasta que el paciente falleciera.

La mortalidad por cáncer de próstata no fue significativamente menor en los pacientes sometidos a prostatectomía radical que en los pacientes en observación ( $p=0.06)$. Estos resultados coinciden con una revisión sistemática de la Sociedad Francesa de Radiología Oncológica (Surveillance active du cáncer de la prostate), en donde se señala que los pacientes no se benefician de la cirugía, pues no encontraron diferencias estadísticamente significativas entre esta terapia y la vigilancia activa. ${ }^{5}$

De igual manera coinciden con el estudio de Hamdy y su grupo, ${ }^{6}$ en el que compararon la vigilancia activa, prostatectomía radical y radioterapia de haz externo para el tratamiento del cáncer de próstata clínicamente localizado ( $\mathrm{n}=82,429$ hombres de 50 a 69 años de edad) en pacientes a quienes se realizó la prueba de antígeno prostático específico. Entre sus resultados, 2664 pacientes fueron diagnosticados con cáncer de próstata localizado, 1643 aceptaron someterse a vigilancia activa, cirugía o radioterapia, con mediana de 10 años de seguimiento. La mortalidad específica por cáncer de próstata fue baja, independientemente del tratamiento asignado, sin observar diferencias significativas entre los tratamientos.

De acuerdo con el estudio de Wilt y sus coautores,${ }^{4}$ la incidencia acumulada de mortalidad a 19.5 años fue de $61.3 \%$ en los pacientes asignados a prostatectomía radical y de $66.8 \%$ a observación. La mediana de supervivencia fue de 13 años en los pacientes con cirugía y de 12.4 años en quienes se asignaron a observación.

Hubo progresión de la enfermedad en $40.9 \%$ de los sujetos asignados a cirugía versus $68.4 \%$ a observación. Estos datos difieren de lo publicado por la Sociedad Francesa de Radiología Oncológica, quienes reportaron que más de $70 \%$ de los tumores de próstata de riesgo bajo o intermedio no mostraron cambio alguno a 10 años de seguimiento. Sin embargo, el estudio de Hamdy y sus colaboradores señaló que la cirugía y la radioterapia se asocian con menor incidencia de evolución de la enfermedad y metástasis que la vigilancia activa; además, estimaron que 27 pacientes requirieron prostatectomía, para evitar que un paciente tuviera metástasis, y 33 recibieron radioterapia en lugar de vigilancia activa, para evitar 1 paciente con enfermedad metastásica.

Se encontraron diferencias importantes a largo plazo en incontinencia urinaria y disfunción eréctil y sexual a favor de los pacientes que solo permanecieron en observación.

La Sociedad Francesa de Radiología Oncológica recomienda la vigilancia activa en pacientes con riesgo bajo de evolución de la enfermedad, pues evita el exceso de procedimientos y tratamientos, sin afectar la seguridad carcinógena, donde la selección de pacientes es decisiva; por tanto, sugiere establecer criterios de selección y progresión a corto plazo, mediante la integración de estudios de imagen y determinación de biomarcadores.

El tratamiento del cáncer de próstata debe individualizarse, tomando en cuenta la edad, clasificación de la enfermedad, concentración de antígeno prostático específico y expectativa de vida del paciente a largo plazo. 


\section{REFERENCIAS}

1. Álvarez-Blanco MA, Escudero-de los Ríos PM, HernándezToríz N. Cáncer de próstata. Artículo de Revisión. Rev Mex Urol 2008;68(4):250-259.

2. Estadísticas importantes sobre el cáncer de próstata. [En línea]. Dirección URL: <https://www.cancer.org/es/cancer/ cancer-de-prostata/acerca/estadisticas-clave.html>. (Consulta: 13 de octubre de 2017).

3. Secretaria de Salud Pública. Programa de Acción: cancer de próstata (2001). 1a Ed.
4. Wilt JT, Jones KM, Barry MJ, et al. Follow-up of prostatectomy versus observation for early prostate cancer. $\mathrm{N}$ Engl Med. 2017;377(2):132-142.

5. Ploussarda G, Hennequinb $C$, Rozet $F$. Surveillance active du cancer de la prostate. Cancer Radiother 2017;21(67):437-441.

6. Hamdy FC, Donovan JL, Lane JA, et al. 10-Year Outcomes after Monitoring, Surgery, or Radiotherapy for Localized Prostate Cancer. N Engl J Med 2016;375:1415-1424.

\section{AVISO PARA LOS AUTORES}

Revista Mexicana de Urología tiene una nueva plataforma de gestión para envío de artículos: https://www.revisionporpares.com/index.php/RMUrol ahí podrá inscribirse a la base de datos administrada por el sistema Open Journal System (OJS) que ofrece las siguientes ventajas para los autores:

- Subir sus artículos directamente al sistema.

- Conocer, en cualquier momento, el estado de los artículos enviados, es decir, si ya fueron asignados a un revisor, aceptados con o sin cambios, o rechazados.

- Participar en el proceso editorial corrigiendo y modificando sus artículos hasta su aceptación final. 